\title{
Cost Per Responder Associated with Biologic Therapies for Crohn's Disease, Psoriasis, and Rheumatoid Arthritis
}

Yifei Liu · Eric Q. Wu • Arielle G. Bensimon · Chun-Po Steve Fan · Yanjun Bao · Arijit Ganguli · Mei Yang · Mary Cifaldi · Parvez Mulani

To view enhanced content go to www.advancesintherapy.com Received: June 14, 2012 / Published online: July 27, 2012

(C) The Author(s) 2012. This article is published with open access at Springerlink.com

\section{ABSTRACT}

Introduction: Biologic therapies have demonstrated efficacy and safety in several chronic systemic disorders. The authors indirectly compared response rates and costs per responder associated with biologic treatments for moderate-to-severe Crohn's disease (CD), psoriasis (Ps), and/or rheumatoid arthritis (RA).

Methods: A systematic literature search was performed to identify phase 3 randomized controlled trials of biologics for CD (adalimumab, infliximab), Ps (adalimumab, etanercept,

\section{Y. Liu $(\bowtie)$}

Division of Pharmacy Practice and Administration, The University of Missouri - Kansas City School of Pharmacy, 2464 Charlotte Street, Kansas City, Missouri 64108, USA

e-mail: liuyif@umkc.edu

E. Q. Wu • A. G. Bensimon - C. P. S. Fan

Analysis Group, Inc., Boston, MA, USA

Y. Bao · A. Ganguli · M. Yang · M. Cifaldi · P. Mulani Abbott Laboratories, Inc., Abbott Park, IL, USA

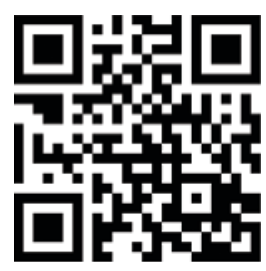

Enhanced content for Advances in Therapy articles is available on the journal web site: www.advancesintherapy.com infliximab, ustekinumab $45 \mathrm{mg}$, ustekinumab $90 \mathrm{mg}$ ), or methotrexate-refractory RA (abatacept, adalimumab, certolizumab, etanercept, golimumab, infliximab, rituximab, tocilizumab). Food and Drug Administration-approved dosing schedules were evaluated. Published response rates were extracted, with response defined in $\mathrm{CD}$, Ps, and RA as: $\geq 70$-point reduction in $\mathrm{CD}$ Activity Index at 12 months; $\geq 75 \%$ improvement in Psoriasis Area and Severity Index at 3 months; and $\geq 50 \%$ improvement in American College of Rheumatology component scores at 6 months. Within each indication, mixed-treatment comparison meta-analyses were conducted to derive pooled estimates and 95\% CIs of response rate difference versus placebo for each biologic, adjusting for cross-trial variation in control-arm response rates. Cost per responder was estimated for each biologic as projected per patient drug costs (2011 US\$) divided by response rate difference.

Results: Altogether, 23 publications were selected. In CD, 12-month cost per responder was estimated at $\$ 116,291$ (95\% CI $\$ 71,637, \$ 208,348)$ for adalimumab and $\$ 125,169$ (95\% CI $\$ 60,532$, $\$ 267,101)$ for infliximab. Among biologics approved in Ps, 3-month cost per responder was lowest for adalimumab $(\$ 9,756 ; 95 \%$ CI $\$ 8,668, \$ 11,131)$, infliximab (\$12,828; 95\% CI \$11,772, \$13,922), and 
ustekinumab $45 \mathrm{mg}(\$ 13,821 ; 95 \%$ CI $\$ 12,599$, $\$ 15,167)$. In RA, biologics with the lowest 6-month cost per responder were adalimumab $(\$ 27,853 ; 95 \%$ CI $\$ 19,284, \$ 40,270)$, etanercept $(\$ 29,140 ; 95 \%$ CI $\$ 14,170, \$ 61,030)$, and tocilizumab $(\$ 31,363 ; 95 \%$ CI $\$ 14,713, \$ 64,232)$.

Conclusion: Meta-analyses of clinical trials found considerable variation in cost-effectiveness of biologic therapies for CD, Ps, and RA. These results may help determine biologic utilization in these chronic diseases.

Keywords: Biologic therapy; Cost-effectiveness; Cost per remitter; Cost per responder; Crohn's disease; Psoriasis; Rheumatoid arthritis

\section{INTRODUCTION}

Since their introduction, targeted biologic therapies have demonstrated efficacy and safety in several chronic systemic disorders, with indications in gastroenterology, dermatology, and rheumatology [1]. In the US, multiple biologics are Food and Drug Administrationapproved for the treatment of Crohn's disease (CD), plaque psoriasis (Ps), and rheumatoid arthritis (RA) in adult patients with moderateto-severe disease. CD, an inflammatory bowel disorder affecting approximately $0.2 \%$ of the population [2], is typically characterized by recurring exacerbations of abdominal pain, diarrhea, fever, and weight loss [3]. Ps is a common, debilitating autoimmune disorder that primarily affects the skin and joints, with a prevalence rate of $2.1 \%$ among US adults [4]. Patients with Ps experience physical pain and diminished quality of life due to erythematous plaques on the body surface $[5,6]$. RA, a chronic inflammatory disorder, is prevalent in approximately $1 \%$ of US adults and can result in progressive joint damage and impaired mobility [7].
Within each of these indications, the availability of highly efficacious biologic therapies has vastly improved the clinical management of patients with active disease despite the use of conventional therapies; the set of biologic drugs approved in CD, Ps, and/or RA includes monoclonal antibodies (adalimumab, certolizumab, golimumab, infliximab, rituximab, tocilizumab, ustekinumab) and recombinant fusion proteins (abatacept, etanercept) [1]. However, these are premium-priced products relative to traditional oral medications [8]. The added expenses of biologic drugs highlight the ongoing need for comparative effectiveness studies to optimize decisions about their use. To date, head-to-head randomized clinical trials comparing alternative biologic regimens are limited to trials of etanercept versus ustekinumab in Ps [9] and abatacept versus infliximab in RA [10]. In the absence of direct comparisons, an up-to-date indirect comparison of biologics via mixed treatment comparison (MTC) meta-analyses would be informative.

Based on a comprehensive review of published clinical trials, the present study sought to compare biologic treatments using MTC meta-analyses of studies in CD, Ps, and RA. Specifically, the study compared cost per responder and cost per remitter across different treatments within each disorder, including: adalimumab and infliximab in CD; adalimumab, etanercept, infliximab, and ustekinumab in Ps; and abatacept, adalimumab, certolizumab, etanercept, golimumab, infliximab, rituximab, and tocilizumab in RA. Costs per responder and remitter provide measures of cost-effectiveness that have both clinical and economic significance to payers and physicians.

For biologic drugs approved in two or more of the diseases, the results of the metaanalyses were used to estimate overall costs per responder and remitter across indications. In order to contain biologic drug costs and promote 
optimal prescribing practices, payers need to consider the total expenditure on biologic drugs in combination with their effectiveness across all indications. Different indications typically encompass different dosing schedules; thus, the acquisition costs of biologic drugs can vary substantially by indication. The relative efficacy of biologic therapies is also highly dependent on the indication. However, because of the administrative burden of varying patient cost sharing and drug tier level by indication, it could be challenging for payers to manage biologic drug use when the drug has multiple indications [8]. In a 2005 poll of health plan directors on the expanded use of biologics, approximately 50\% of participants responded that their organization would not be capable of appropriately managing a biologic therapy with multiple indications [8]. In cases where it is not feasible to manage coverage for a particular drug by indication, an analysis of blended cost-effectiveness across indications may provide a rational basis for the formulary management of drugs approved in more than one indication. Accordingly, the authors estimated blended costs per responder and remitter to compare adalimumab and infliximab across the CD, Ps, and RA indications, and to compare adalimumab, etanercept, and infliximab across the Ps and RA indications.

\section{MATERIALS AND METHODS}

\section{Study Inclusion Criteria}

A systematic literature review was performed to identify published randomized, controlled clinical trials of biologic therapies for the treatment of moderate-to-severe CD, Ps, or RA. The search was confined to phase 3 trials that evaluated a biologic treatment in comparison with either placebo or another biologic. Specific trial selection criteria varied by indication to reflect general differences in the time horizon and design of phase 3 biologic drug trials between the three disease areas. Trials of adalimumab and infliximab in CD were included if they followed patients for a minimum of 52 weeks, reported response and remission rates based on the Crohn's Disease Activity Index (CDAI), and featured an induction-only placebo arm (i.e., patients were switched to placebo after receiving an induction regimen of the biologic), which is the usual comparison arm protocol that has been used in phase 3 trials of biologic drugs in CD. Studies of adalimumab, etanercept, infliximab, or ustekinumab for Ps were selected if they followed patients for at least 10 weeks, reported response rates based on the Psoriasis Area and Severity Index (PASI), and included either a placebo or another biologic in the trial. In RA, trials that evaluated methotrexate (MTX) combined with abatacept, adalimumab, certolizumab, etanercept, golimumab, infliximab, rituximab, or tocilizumab were included. Trials were also required to follow patients for a minimum of 24 weeks, report response rates based on the American College of Rheumatology (ACR) score, and feature a comparison arm consisting of MTX combined with either placebo or a different biologic treatment. Additionally, in order to minimize between-study heterogeneity and capture outcomes for the RA patient population most likely to be treated with biologic drugs in real-world clinical settings, the meta-analysis of RA trials was restricted to studies in which patients were required to have previously failed treatment with MTX; studies that enrolled patients who were MTX-naïve or who had previously failed biologic therapy were considered too dissimilar to combine with trials in the MTX-refractory population and were, therefore, excluded from the meta-analysis.

All searches were conducted in the PubMed database; keywords included combinations 
of the disease plus any of the biologic drug names in that indication. Trials registered on ClinicalTrials.gov were also reviewed to check for additional studies.

\section{Collection of Efficacy Data}

Altogether, 23 publications met the selection criteria, including 2 in CD, 10 in Ps, and 11 in RA. For adalimumab and infliximab treatment in the CD indication, the clinical trials of Colombel et al. [11] and Hanauer et al. [12] were selected for inclusion. In both trials, all patients received induction biologic therapy and were then randomized to biologic therapy or placebo based on their initial response status. Initial clinical response was defined as a decrease in CDAI score of $\geq 70$ points from baseline (CR-70), and was assessed at week 4 by Colombel et al. and week 2 by Hanauer et al. Both publications reported subsequent efficacy outcomes among initial CR-70 responders only. For the present metaanalysis, rates of CR-70 response and remission defined as CDAI $<150$ at approximately 1 year (i.e., week 54 or 56) were extracted for the initial responders population; the percentage of enrolled patients who achieved an initial CR-70 response was also collected from either trial.

The selected clinical trials of biologic drugs in Ps followed patients for a period of 10-16 weeks [9, 13-21]. Efficacy results in terms of PASI 75 and PASI 90 response rates, defined respectively as improvements of $\geq 75 \%$ and $\geq 90 \%$ in PASI score from baseline, were extracted from each study. Because remission of Ps is not consistently defined in the literature, PASI 90 response was used as a proxy measure for remission in this indication. Whenever available, week 12 results were collected.

Rates of ACR 50 and ACR 70 response, defined respectively as improvements of $\geq 50 \%$ and $\geq 70 \%$ in the number of both swollen and tender joints and in at least three of five additional domain scores, were collected from clinical trials of biologic drugs in RA [10, 22-31]. Results were extracted for the study visit occurring at approximately 6 months (i.e., week 24-30). In RA, the percentage of patients achieving ACR 50 is the usual outcome used for response assessment; ACR 70 response was selected as a suitable proxy for remission owing to the inconsistent availability of other outcome measures for remission in the included trials.

\section{Statistical Analysis}

\section{MTC Meta-Analysis}

For each indication, meta-analyses were conducted to synthesize efficacy results from the included trials and derive pooled response and remission rates for each biologic regimen. The evidence synthesis method in the York/Woolacott cost-effectiveness model of biologic drugs for psoriasis was adapted for this MTC study [32]. Bayesian logistic regression was applied to analyze the dichotomous outcomes using Markov chain Monte Carlo with Gibbs sampling method by indication. An MTC meta-analysis approach was selected for its ability to synthesize summarylevel clinical evidence from multiple studies while adjusting for between-trial differences in placebo response rates [33-35]; in contrast to standard meta-analyses, this method also allowed for the combination of data from direct comparisons (i.e., trials comparing two different biologic drugs) with indirect evidence from placebo-controlled trials of biologics [36]. Using MTC, the relative efficacy of each biologic therapy was calculated in terms of incremental response/remission rate, defined as the difference in response/remission rates between the therapy and placebo. Based on the posterior distribution of the relative efficacy, the posterior mean was calculated as a point estimate of the relative efficacy, and 95\% CIs were 
approximated using the highest posterior density method. All Bayesian analyses were conducted using R/OpenBUGS software (R Foundation for Statistical Computing).

\section{Number Needed to Treat}

The number needed to treat (NNT) per additional responder/remitter associated with each biologic drug by indication was estimated using the point estimate of relative efficacy. NNT can be interpreted as the number of patients who need to be treated with a particular drug in order to achieve one additional positive outcome (i.e., response, remission) [37]. For each drug evaluated in Ps and RA, NNT per additional responder was calculated as the reciprocal of the incremental response rate versus placebo for that treatment. For drugs assessed in CD, a different formula for NNT was used owing to the design of the phase 3 clinical trials of adalimumab and infliximab for $\mathrm{CD}$, in which results were reported for initial responders only. Specifically, patients with CD without an initial response to adalimumab or infliximab were assumed to discontinue therapy at week 4 and achieve neither response nor remission at 1 year. NNT per additional responder was accordingly estimated as: $1 /$ ([initial response rate] ${ }^{*}$ [incremental response rate vs. placebo at 1 year among initial responders]). The corresponding 95\% CI was approximated by the posterior distribution of the NNT based on the Markov chain Monte Carlo results. Similar calculations were performed for the NNT per additional remitter.

\section{Measurement of Cost-Effectiveness}

Costs per additional responder and remitter were estimated for each biologic drug as the estimated NNT multiplied by the projected drug cost per patient, and corresponding 95\% CIs were estimated. In accordance with the length of the clinical trials included in the meta-analyses, drug acquisition and administration costs were calculated over a time horizon of 52 weeks in CD, 12 weeks in Ps, and 24 weeks in RA. In Ps and RA, costs were estimated by assuming full compliance to the indicated dosages within the specified time frame. In $\mathrm{CD}$, per-patient cost was estimated with the assumption that initial responders had full compliance to the indicated dosages up to week 52, while initial nonresponders only received dosages before the end of week 4.

For biologic drugs approved in multiple indications (adalimumab, etanercept, and infliximab), blended costs per additional responder and remitter were calculated as a weighted average of the estimated costs per additional responder and remitter across indications. To use a standardized time horizon across the three diseases, costs per additional responder/remitter within the Ps and RA indications were first recalculated using a 52 -week time frame, with the assumption that shorter-term response rates were maintained to year-end. Because etanercept is not approved in the CD indication, two separate comparisons were conducted: (i) blended costs per additional responder/remitter for adalimumab versus infliximab across CD, Ps, and RA; and (ii) blended costs per additional responder/remitter for adalimumab versus etanercept versus infliximab across Ps and RA. In either comparison, indication-specific cost per responder/remitter estimates were weighted in the blended average according to the total volume of biologic drug prescriptions written in the US for each indication. Prescription volume was used as an indicator of the size of the biologic market in each disease area, and was estimated based on Wolters Kluwer data in July 2010 (unpublished data), the most recent month of data available at the time of manuscript development.

For all calculations of costs per responder and remitter, US wholesale acquisition costs 
as of January 2011 were used to determine drug acquisition costs (ReadyPrice ${ }^{\circledR}$, Thomson Micromedex, Greenwood Village, Colorado, USA). Recommended dosing schedules based on US labels were assumed for each drug (Table 1). Per-infusion drug cost for abatacept, infliximab, and tocilizumab were calculated based on an average weight of $70 \mathrm{~kg}$ in CD [11] and RA [26], and $90 \mathrm{~kg}$ in Ps [14]. Administration cost per infusion was obtained from Medicare Current Procedural Terminology (CPT) payment information for 2011 (CPT codes 96413 and 96415 for abatacept, infliximab, rituximab, and tocilizumab; CPT code 96401 for certolizumab and ustekinumab). Drug acquisition and administration costs were prorated in order to obtain total costs over the specified time horizon within each indication.

\section{RESULTS}

\section{Meta-Analysis Results in CD}

In the included phase 3 clinical trials of both adalimumab and infliximab, the proportion of patients who achieved an initial response to biologic induction therapy constituted $58 \%$ of the overall trial population. The MTC meta-analysis performed in the $\mathrm{CD}$ indication analyzed response and remission rates to adalimumab and infliximab within the initial responder population from either trial. Results from the meta-analysis indicated that, among initial responders, the incremental CR-70 response rate (i.e., the difference in response rates between treatment vs. induction-only placebo) was $24.7 \%$ (95\% CI 12.6\%, 37.5\%) for adalimumab and 20.8\% (95\% CI 7.7\%, 34.9\%) for infliximab (Table 2). Adalimumab (23.6\%; 95\% CI 10.4\%, 38.1\%) also had a higher incremental remission rate compared to infliximab (14.9\%; 95\% CI 2.6\%, 27.2\%) (Table 2).

Table 3 presents the NNTs and 52-week costs per additional responder and remitter associated with either biologic in $\mathrm{CD}$, in which initial nonresponders were assumed to have failed treatment and discontinued biologic use by week 4. In CD, 12-month cost per responder was estimated at $\$ 116,291$ (95\% CI \$71,637, \$208,348) for adalimumab and $\$ 125,169$ (95\% CI $\$ 60,532$, $\$ 267,101)$ for infliximab. Compared to infliximab, adalimumab was associated with reductions of $\$ 8,878$ in cost per additional responder and $\$ 52,983$ in cost per additional remitter.

\section{Meta-Analysis Results in Ps}

In the MTC meta-analysis of biologic trials in Ps, incremental PASI 75 response rates relative to placebo were highest for infliximab (74.9\%) and ustekinumab $90 \mathrm{mg}$ (67.9\%) (Table 4). Adalimumab (64.4\%) and ustekinumab $45 \mathrm{mg}$ (62.7\%) had comparable incremental response rates, while etanercept showed the lowest response probability versus placebo (47.0\%). The relative efficacy of the comparator drugs in terms of remission, assessed based on PASI 90 response, showed a similar pattern.

Over the 12-week time horizon, adalimumab was associated with the lowest cost per additional responder $(\$ 9,756 ; 95 \%$ CI $\$ 8,668$, $\$ 11,131)$ among the biologics in Ps, followed by infliximab (\$12,828; 95\% CI \$11,772, \$13,922), ustekinumab $45 \mathrm{mg}(\$ 13,821 ; 95 \%$ CI $\$ 12,599$, $\$ 15,167)$, etanercept $(\$ 21,770 ; 95 \%$ CI $\$ 19,231$, $\$ 24,644)$, and ustekinumab $90 \mathrm{mg}(\$ 25,327 ; 95 \%$ CI $\$ 23,372, \$ 27,332$ ) (Table 5). Results were similar with respect to costs per additional remitter.

\section{Meta-Analysis Results in RA}

Based on the meta-analysis of trials among patients with MTX-refractory RA, the biologics with the highest incremental ACR 50 response probabilities relative to placebo were adalimumab (36.1\%), etanercept (35.1\%), and 
certolizumab (33.3\%) (Table 6). Incremental remission probabilities based on ACR 70 were highest for adalimumab (21.1\%), certolizumab (19.8\%), and etanercept (16.4\%).

Cost per additional responder over 24 weeks was lowest for adalimumab $(\$ 27,853 ; 95 \%$ CI $\$ 19,284$, $\$ 40,270)$, followed by etanercept $(\$ 29,140 ; 95 \%$ CI $\$ 14,170, \$ 61,030)$, tocilizumab $(\$ 31,363 ; 95 \%$ CI $\$ 14,713, \$ 64,232)$, and certolizumab $(\$ 34,979$; 95\% CI \$23,636, \$51,166) (Table 7); higher costs per responder were estimated for rituximab, abatacept, infliximab, and golimumab. The lowest costs per additional remission were estimated for adalimumab $(\$ 47,533 ; 95 \%$ CI $\$ 23,939, \$ 86,730)$ and tocilizumab $(\$ 48,320 ; 95 \%$ CI $\$ 15,766$, $\$ 135,922)$.

\section{Cross-Indication Results}

In the calculation of cross-indication 1-year costs per additional responder/remitter, the indicationspecific results in $\mathrm{CD}$, Ps, and RA were weighted in a ratio of 1:1.02:5.07 according to the relative volume of prescriptions written for biologics in each disease area. The weighted average 1-year cost per additional responder across the Ps and RA indications was \$56,219 (95\% CI \$40,592, \$78,426) for adalimumab, $\$ 62,283$ (95\% CI $\$ 34,815$, $\$ 119,476)$ for etanercept, and $\$ 82,683$ (95\% CI $\$ 46,082, \$ 146,609$ ) for infliximab (Table 8). Blended 1-year cost per additional remitter in Ps and RA was lower for adalimumab by $\$ 38,445$ compared to etanercept and by $\$ 40,101$ compared to infliximab. Across all three indications, adalimumab was associated with $\$ 23,984$ and $\$ 41,919$ lower 1-year costs per additional responder and remitter relative to infliximab, respectively (Table 9).

\section{DISCUSSION}

This study used MTC meta-analyses of published clinical trials to evaluate the cost per responder and cost per remitter associated with different biologic therapies approved for the treatment of CD, Ps, and RA in the US. In addition to comparing biologic drugs for each indication, the study estimated cross-indication 1-year costs per responder/remitter for biologics approved in at least two of the three indications studied (i.e., adalimumab, etanercept, and infliximab). Consistent with a previous cost-effectiveness study of biologics for moderately-to-severely active CD in the US [38], adalimumab was associated with lower 1-year costs per responder and remitter compared to infliximab within the CD indication. Adalimumab also had the lowest 12-week costs per responder and remitter of the biologics assessed in Ps, followed by infliximab $5 \mathrm{mg} / \mathrm{kg}$ and ustekinumab $45 \mathrm{mg}$; these results echoed findings from a previous cost-effectiveness study conducted by Schmitt-Rau et al. [39] in moderateto-severe Ps. Among the biologics evaluated in RA, adalimumab had the lowest 24-week cost per responder, followed by etanercept, tocilizumab, and certolizumab. RA drugs with the lowest cost per remitter were adalimumab and tocilizumab. Consistent with indication-specific results, adalimumab demonstrated lower 1-year costs per additional responder and remitter versus etanercept and infliximab in Ps and RA, and versus infliximab in CD, Ps, and RA.

Overall, cost-effectiveness varied substantially across biologics, particularly for Ps and RA. In Ps, 12-week costs per responder and remitter were more than twice as high for etanercept and ustekinumab $90 \mathrm{mg}$ than adalimumab. In $\mathrm{RA}$, rituximab, abatacept, and infliximab had notably higher costs per responder and remitter compared to other drugs evaluated. The present analysis considers only biologic drug acquisition and infusion costs and does not capture additional medical expenses associated with treatment failure, such as hospitalization costs. Nonetheless, the wide variation in costs 

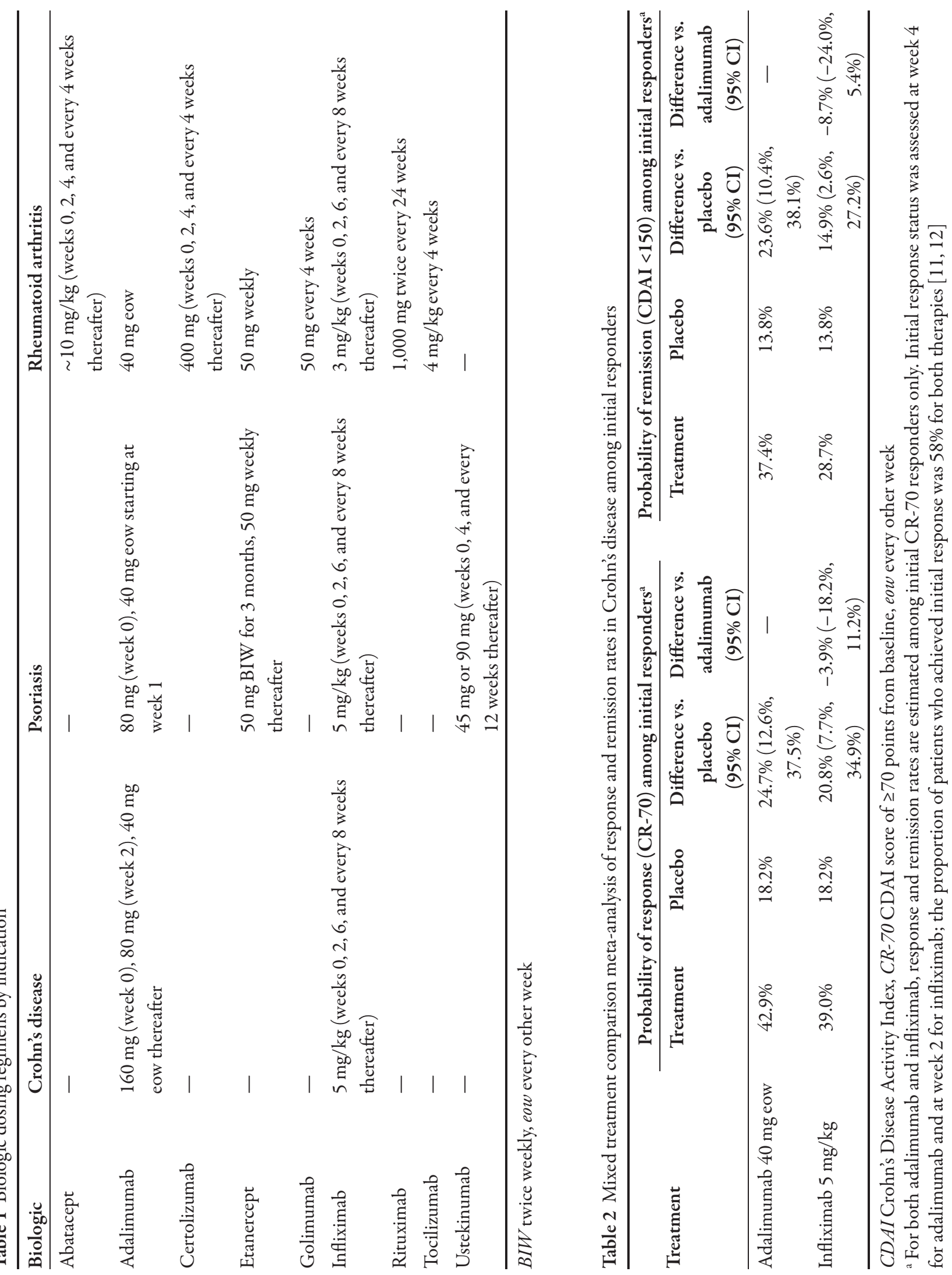


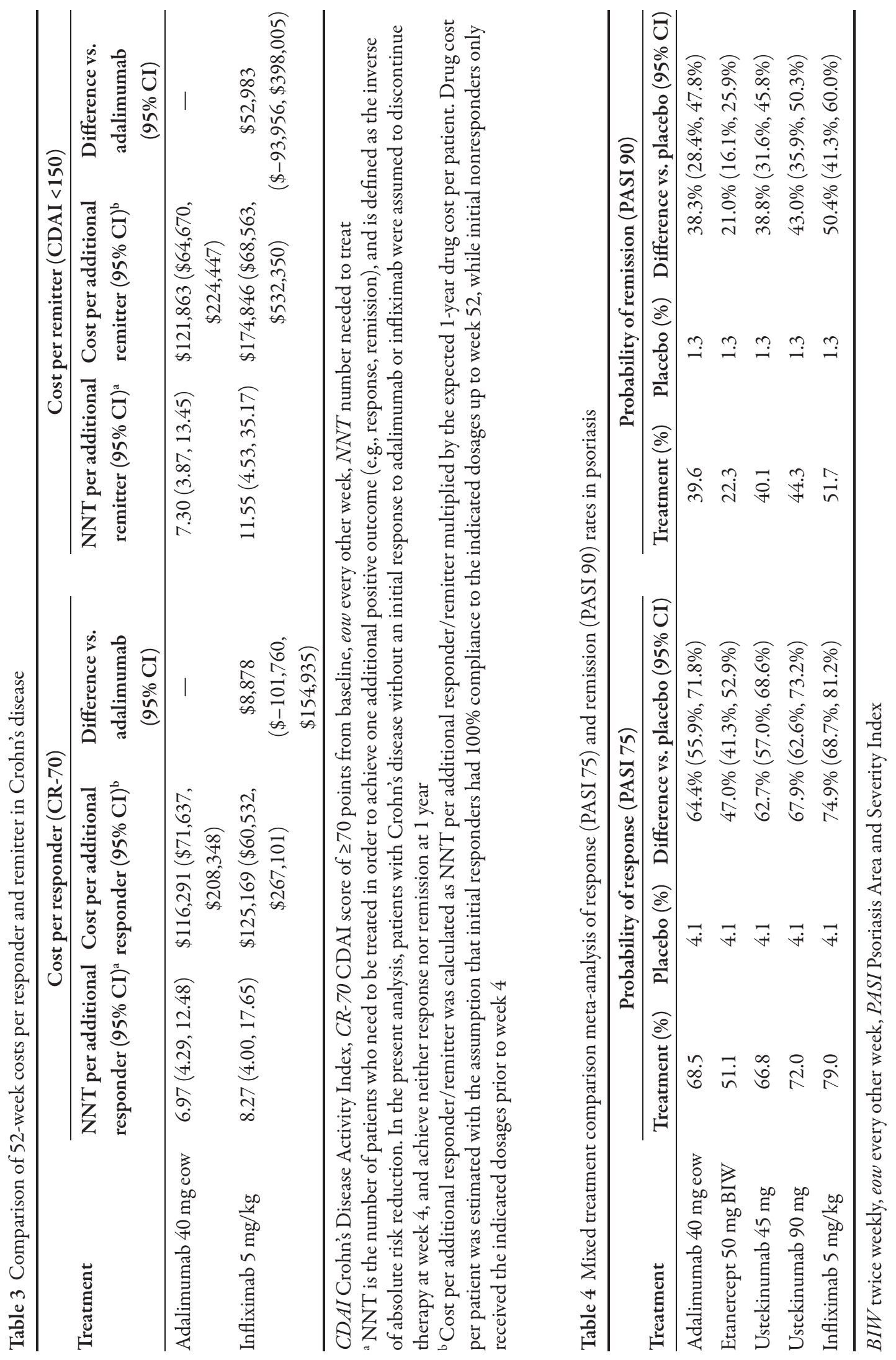



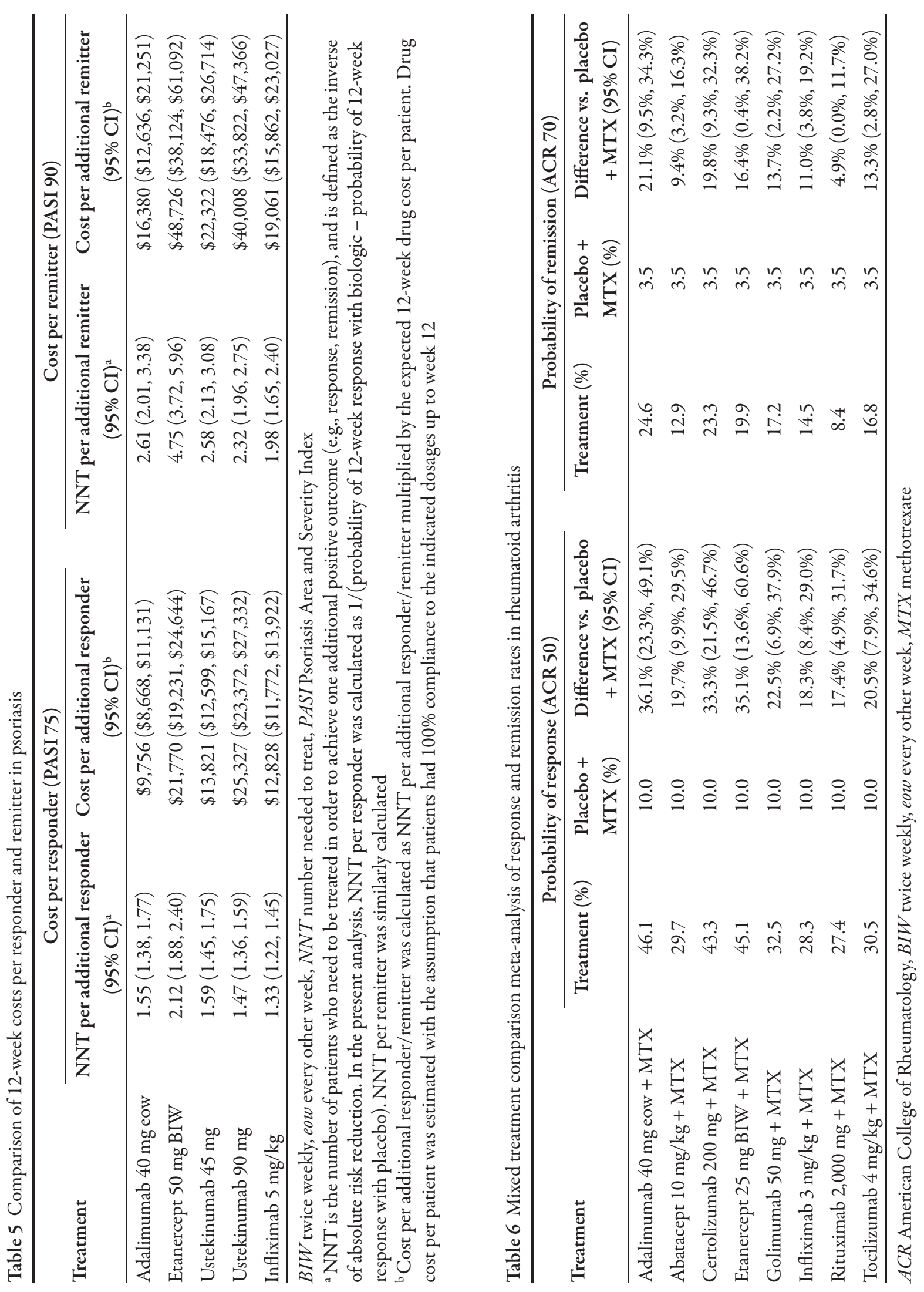

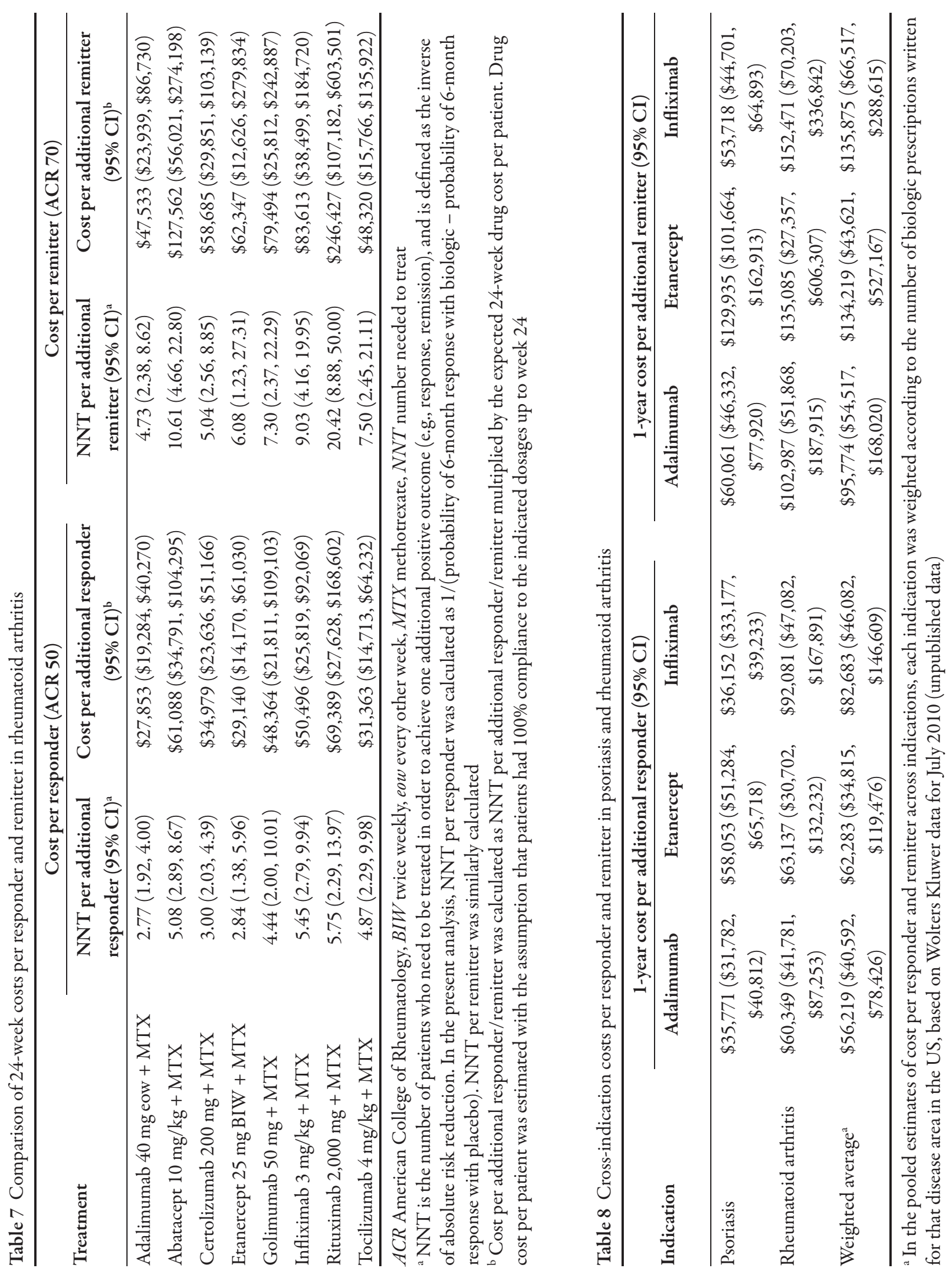
Table 9 Cross-indication costs per responder and remitter in Crohn's disease, psoriasis, and rheumatoid arthritis

\begin{tabular}{|c|c|c|c|c|}
\hline \multirow[b]{2}{*}{ Indication } & \multicolumn{2}{|c|}{ 1-year cost per additional responder $(95 \% \mathrm{CI})$} & \multicolumn{2}{|c|}{ 1-year cost per additional remitter $(95 \% \mathrm{CI})$} \\
\hline & Adalimumab & Infliximab & Adalimumab & Infliximab \\
\hline Crohn's disease & $\begin{array}{c}\$ 116,291(\$ 71,637 \\
\$ 208,348)\end{array}$ & $\begin{array}{c}\$ 125,169(\$ 60,532 \\
\$ 267,101)\end{array}$ & $\begin{array}{c}\$ 121,863(\$ 64,670 \\
\$ 224,447)\end{array}$ & $\begin{array}{c}\$ 174,846(\$ 68,563, \\
\$ 532,350)\end{array}$ \\
\hline Psoriasis & $\begin{array}{c}\$ 35,771(\$ 31,782 \\
\$ 40,812)\end{array}$ & $\begin{array}{c}\$ 36,152(\$ 33,177, \\
\$ 39,233)\end{array}$ & $\begin{array}{c}\$ 60,061(\$ 46,332, \\
\$ 77,920)\end{array}$ & $\begin{array}{c}\$ 53,718(\$ 44,701 \\
\$ 64,893)\end{array}$ \\
\hline Rheumatoid arthritis & $\begin{array}{c}\$ 60,349(\$ 41,781, \\
\$ 87,253)\end{array}$ & $\begin{array}{c}\$ 92,081(\$ 47,082, \\
\$ 167,891)\end{array}$ & $\begin{array}{c}\$ 102,987(\$ 51,868 \\
\$ 187,915)\end{array}$ & $\begin{array}{c}\$ 152,471(\$ 70,203, \\
\$ 336,842)\end{array}$ \\
\hline Weighted average ${ }^{\mathrm{a}}$ & $\begin{array}{c}\$ 64,691(\$ 47,377, \\
\$ 91,780)\end{array}$ & $\begin{array}{c}\$ 88,675(\$ 53,500, \\
\$ 160,856)\end{array}$ & $\begin{array}{c}\$ 99,453(\$ 60,594, \\
\$ 174,312)\end{array}$ & $\begin{array}{c}\$ 141,372(\$ 72,967, \\
\$ 316,252)\end{array}$ \\
\hline
\end{tabular}

${ }^{\text {a }}$ In the pooled estimates of cost per responder and remitter across indications, each indication was weighted according to the number of biologic prescriptions written for that disease area in the US, based on Wolters Kluwer data for July 2010 (unpublished data)

per responder and remitter across biologics demonstrates the potential usefulness of comparative effectiveness research in informing treatment decisions and formulary placement in these three disease areas.

To the authors' knowledge, the present study is the first to evaluate the blended costeffectiveness of biologics across indications. As exemplified by cost-effectiveness results for adalimumab, etanercept, and infliximab across their indications, a biologic therapy approved in multiple indications is likely to be associated with varying clinical efficacy, dosing regimens, and acquisition costs depending on the disease. Thus, in the context of expanded indications for biologics, payers must identify and implement feasible strategies for promoting appropriate utilization of biologics within each disease area; such strategies may aim to stratify drug coverage by patient population. In the meantime, information on the cross-indication costeffectiveness of biologics may assist formulary decision-making by facilitating the comparison of therapies approved for the same set of diseases.

Because CD, Ps, and RA are chronic disorders, the cost-effectiveness of biologic therapies for these indications should ideally be assessed over long time horizons. Thus, one limitation of this study is the paucity of long-term efficacy data available for the evaluation of costs per responder/remitter within the Ps and RA indications. The clinical efficacies of biologics are well-reported during the first 3 months of treatment in patients with moderate-to-severe Ps $[9,13-21]$, and during the first 6 months of therapy in patients with moderateto-severe, methotrexate-refractory RA [10, 22-31]; however, few randomized, double-blinded, placebocontrolled trials have documented the benefits of biologics over longer time frames in these patient populations. The analysis of 1-year cross-indication costs per responder/remitter, therefore, assumed that short-term response rates in Ps and RA were maintained to year-end, similar to the approach used in previous cost-effectiveness analyses of biologics [32, 35].

In this study, the efficacy of different biologic drugs was compared using a MTC meta-analysis of clinical trials. While clinical trials provide unbiased comparisons of clinical efficacy in a controlled environment, the strict treatment protocols and eligibility criteria used in such studies may not be representative of actual clinical practice. For example, differing levels of compliance and persistence to biologic therapies may be a more 
important determinant of treatment outcomes in real-world practice than in clinical trial settings. Another limitation of this analysis is the possibility of confounding due to patient heterogeneity across clinical trials. By adjusting for variations in comparison arm response rates across the included trials, MTC meta-analysis was expected to result in less biased comparisons of clinical efficacy between different biologics. However, this metaanalytic method may not fully adjust for cross-trial heterogeneity, particularly if the magnitude of the biologic treatment effect versus the comparison arm varies depending on the patient population's baseline characteristics [40]. Additional headto-head clinical trials of biologics would be required to eliminate the potential for unobserved confounding.

\section{CONCLUSION}

This meta-analytic study found considerable range in estimates of costs per additional responder and remitter across different biologics indicated for moderate-to-severe CD, Ps, and RA. Moreover, the relative efficacy of biologic agents varied depending on the indication, which highlights the need for health plans to consider overall cost-effectiveness across indications for biologics approved in multiple diseases. In the cross-indication analysis, adalimumab was associated with lower blended costs per responder and remitter compared to other biologics approved in two or more of the diseases studied, including etanercept and infliximab. Results suggest the potential for biologics to be used more cost-effectively in these chronic disease areas.

\section{ACKNOWLEDGMENTS}

Funding and article processing charges for this research were provided by Abbott Laboratories, Abbott Park, Illinois, USA. Dr. Liu is the guarantor for this article, and takes responsibility for the integrity of the work as a whole.

Conflict of Interest. Yanjun Bao, Arijit Ganguli, Mei Yang, Mary Cifaldi, and Parvez Mulani are employees of Abbott Laboratories, Abbott Park, IL, USA. Eric Q. Wu, Arielle G. Bensimon, and Chun-Po Steve Fan are employees of Analysis Group, Boston, MA, USA, which received funding for this study.

Open Access. This article is distributed under the terms of the Creative Commons Attribution Noncommercial License which permits any noncommercial use, distribution, and reproduction in any medium, provided the original author(s) and source are credited.

\section{REFERENCES}

1. Gartlehner G, Thieda P, Morgan LC, Thaler K, Hansen RA, Jonas B. Drug Class Review: Targeted Immune Modulators: Final Report Update 2. Portland, OR: Oregon Health \& Science University; 2009.

2. Kappelman MD, Rifas-Shiman SL, Kleinman K, et al. The prevalence and geographic distribution of Crohn's disease and ulcerative colitis in the United States. Clin Gastroenterol Hepatol. 2007;5:1424-9.

3. Loftus EV Jr, Schoenfeld P, Sandborn WJ. The epidemiology and natural history of Crohn's disease in population-based patient cohorts from North America: a systematic review. Aliment Pharmacol Ther. 2002;16:51-60.

4. National Psoriasis Foundation. Benchmark survey on psoriasis and psoriatic arthritis: summary of top-line results. Available at: http://www.psoriasis.org/files/pdfs/ press/npfsurvey.pdf. Accessed December 15, 2010.

5. Kimball AB, Jacobson C, Weiss S, Vreeland MG, Wu Y. The psychosocial burden of psoriasis. Am J Clin Dermatol. 2005:6:383-92.

6. Rapp SR, Feldman SR, Exum ML, Fleischer AB Jr, Reboussin DM. Psoriasis causes as much disability as other major medical diseases. J Am Acad Dermatol. 1999;41:401-7.

7. Helmick CG, Felson DT, Lawrence RC, et al; National Arthritis Data Workgroup. Estimates of the prevalence of arthritis and other rheumatic conditions in the United States: Part I. Arthritis Rheum. 2008;58:15-25. 
8. Cohen M, Morrow T, Penna P. Managing the expanded use of biologics across therapeutic areas: an example from B-cell targeted therapies. Am J Manag Care. 2006;12:S24-37.

9. Griffiths CE, Strober BE, van de Kerkhof P, et al; ACCEPT Study Group. Comparison of ustekinumab and etanercept for moderate-to-severe psoriasis. N Engl J Med. 2010;362:118-28.

10. Schiff M, Keiserman M, Codding C, et al. Efficacy and safety of abatacept or infliximab vs placebo in ATTEST: a phase III, multi-centre, randomised, double-blind, placebo-controlled study in patients with rheumatoid arthritis and an inadequate response to methotrexate. Ann Rheum Dis. 2008;67:1096-103.

11. Colombel JF, Sandborn WJ, Rutgeerts P, et al. Adalimumab for maintenance of clinical response and remission in patients with Crohn's disease: the CHARM trial. Gastroenterology. 2007;132:52-65.

12. Hanauer SB, Feagan BG, Lichtenstein GR, et al; ACCENT I Study Group. Maintenance infliximab for Crohn's disease: the ACCENT I randomised trial. Lancet. 2002;359:1541-9.

13. Saurat JH, Stingl G, Dubertret L, et al; CHAMPION Study Investigators. Efficacy and safety results from the randomized controlled comparative study of adalimumab vs. methotrexate vs. placebo in patients with psoriasis (CHAMPION). Br J Dermatol. 2008;158:558-66.

14. Menter A, Tyring SK, Gordon K, et al. Adalimumab therapy for moderate to severe psoriasis: a randomized, controlled phase III trial. J Am Acad Dermatol. 2008;58:106-15.

15. Papp KA, Tyring S, Lahfa M, et al; Etanercept Psoriasis Study Group. A global phase III randomized controlled trial of etanercept in psoriasis: safety, efficacy, and effect of dose reduction. Br J Dermatol. 2005;152:1304-12.

16. Leonardi CL, Powers JL, Matheson RT, et al; Etanercept Psoriasis Study Group. Etanercept as monotherapy in patients with psoriasis. N Engl J Med. 2003;349:2014-22.

17. Tyring S, Gordon KB, Poulin Y, et al. Long-term safety and efficacy of $50 \mathrm{mg}$ of etanercept twice weekly in patients with psoriasis. Arch Dermatol. 2007;143:719-26.

18. Reich K, Nestle FO, Papp K, et al; EXPRESS study investigators. Infliximab induction and maintenance therapy for moderate-to-severe psoriasis: a phase III, multicentre, double-blind trial. Lancet. 2005;366:1367-74.
19. Menter A, Feldman SR, Weinstein GD, et al. A randomized comparison of continuous vs. intermittent infliximab maintenance regimens over 1 year in the treatment of moderate-tosevere plaque psoriasis. J Am Acad Dermatol. 2007;56:31.e1-15.

20. Leonardi CL, Kimball AB, Papp KA, et al; PHOENIX 1 study investigators. Efficacy and safety of ustekinumab, a human interleukin-12/23 monoclonal antibody, in patients with psoriasis: 76-week results from a randomised, double-blind, placebo-controlled trial (PHOENIX 1). Lancet. 2008;371:1665-74.

21. Papp KA, Langley RG, Lebwohl M, et al; PHOENIX 2 study investigators. Efficacy and safety of ustekinumab, a human interleukin-12/23 monoclonal antibody, in patients with psoriasis: 52-week results from a randomised, double-blind, placebo-controlled trial (PHOENIX 2). Lancet. 2008;371:1675-84.

22. Kremer JM, Genant HK, Moreland LW, et al. Effects of abatacept in patients with methotrexateresistant active rheumatoid arthritis: a randomized trial. Ann Intern Med. 2006;144:865-76.

23. Keystone EC, Kavanaugh AF, Sharp JT, et al. Radiographic, clinical, and functional outcomes of treatment with adalimumab (a human antitumor necrosis factor monoclonal antibody) in patients with active rheumatoid arthritis receiving concomitant methotrexate therapy: a randomized, placebo-controlled, 52-week trial. Arthritis Rheum. 2004;50:1400-11.

24. Keystone E, Heijde D, Mason D Jr, et al. Certolizumab pegol plus methotrexate is significantly more effective than placebo plus methotrexate in active rheumatoid arthritis: findings of a fifty-two-week, phase III, multicenter, randomized, double-blind, placebocontrolled, parallel-group study. Arthritis Rheum. 2008;58:3319-29.

25. Keystone EC, Genovese MC, Klareskog L, et al; GOFORWARD Study. Golimumab, a human antibody to tumour necrosis factor \{alpha\} given by monthly subcutaneous injections, in active rheumatoid arthritis despite methotrexate therapy: the GOFORWARD Study. Ann Rheum Dis. 2009;68:789-96.

26. Smolen JS, Beaulieu A, Rubbert-Roth A, et al; OPTION Investigators. Effect of interleukin-6 receptor inhibition with tocilizumab in patients with rheumatoid arthritis (OPTION study): a double-blind, placebo-controlled, randomised trial. Lancet. 2008;371:987-97. 
27. Smolen J, Landewé RB, Mease P, et al. Efficacy and safety of certolizumab pegol plus methotrexate in active rheumatoid arthritis: the RAPID 2 study. A randomised controlled trial. Ann Rheum Dis. 2009;68:797-804.

28. Weinblatt ME, Kremer JM, Bankhurst AD, et al. A trial of etanercept, a recombinant tumor necrosis factor receptor:Fc fusion protein, in patients with rheumatoid arthritis receiving methotrexate. N Engl J Med. 1999;340:253-9.

29. Weinblatt ME, Keystone EC, Furst DE, et al. Adalimumab, a fully human anti-tumor necrosis factor alpha monoclonal antibody, for the treatment of rheumatoid arthritis in patients taking concomitant methotrexate: the ARMADA trial. Arthritis Rheum. 2003;48:35-45.

30. Emery P, Deodhar A, Rigby WF, et al. Efficacy and safety of different doses and retreatment of rituximab: a randomised, placebo-controlled trial in patients who are biological naive with active rheumatoid arthritis and an inadequate response to methotrexate (Study Evaluating Rituximab's Efficacy in MTX iNadequate rEsponders [SERENE]). Ann Rheum Dis. 2010;69:1629-35.

31. Maini R, St Clair EW, Breedveld F, et al. Infliximab (chimeric anti-tumour necrosis factor alpha monoclonal antibody) versus placebo in rheumatoid arthritis patients receiving concomitant methotrexate: a randomised phase III trial. ATTRACT Study Group. Lancet. 1999;354:1932-9.

32. Woolacott N, Hawkins N, Mason A, et al. Etanercept and efalizumab for the treatment of psoriasis: a systematic review. Health Technol Assess. 2006;10:1-233, i-iv.
33. Nixon RM, Bansback N, Brennan A. Using mixed treatment comparisons and meta-regression to perform indirect comparisons to estimate the efficacy of biologic treatments in rheumatoid arthritis. Stat Med. 2007;26:1237-54.

34. Kristensen LE, Christensen R, Bliddal H, Geborek P, Danneskiold-Samsøe B, Saxne T. The number needed to treat for adalimumab, etanercept, and infliximab based on ACR50 response in three randomized controlled trials on established rheumatoid arthritis: a systematic literature review. Scand J Rheumatol. 2007;36:411-7.

35. Sizto S, Bansback N, Feldman SR, Willian MK, Anis AH. Economic evaluation of systemic therapies for moderate to severe psoriasis. Br J Dermatol. 2009; 160:1264-72.

36. Lu G, Ades AE. Combination of direct and indirect evidence in mixed treatment comparisons. Stat Med. 2004;23:3105-24.

37. Laupacis A, Sackett DL, Roberts RS. An assessment of clinically useful measures of the consequences of treatment. N Engl J Med. 1988;318:1728-33.

38. Yu AP, Johnson S, Wang ST, et al. Cost utility of adalimumab versus infliximab maintenance therapies in the United States for moderately to severely active Crohn's disease. Pharmacoeconomics. 2009;27:609-21.

39. Schmitt-Rau K, Rosenbach T, Radtke MA, Augustin M. Cost-effectiveness of biological therapy in remission induction of moderate to severe plaque psoriasis. Dermatology. 2010;221:236-42.

40. Bucher HC, Guyatt GH, Griffith LE, Walter SD. The results of direct and indirect treatment comparisons in meta-analysis of randomized controlled trials. J Clin Epidemiol. 1997;50:683-91. 